\title{
BOUNDED SOLUTIONS OF NONLINEAR CAUCHY PROBLEMS
}

\author{
JOSEF KREULICH
}

Received 28 February 2001

For a given closed and translation invariant subspace $Y$ of the bounded and uniformly continuous functions, we will give criteria for the existence of solutions $u \in Y$ to the equation $u^{\prime}(t)+A(u(t))+\omega u(t) \ni f(t), t \in \mathbb{R}$, or of solutions $u$ asymptotically close to $Y$ for the inhomogeneous differential equation $u^{\prime}(t)+A(u(t))+\omega u(t) \ni f(t), t>0, u(0)=u_{0}$, in general Banach spaces, where $A$ denotes a possibly nonlinear accretive generator of a semigroup. Particular examples for the space $Y$ are spaces of functions with various almost periodicity properties and more general types of asymptotic behavior.

\section{Introduction}

For the case of linear Cauchy problems, results on the asymptotic behavior of the solutions can be obtained by applying the representation formula

$$
u(t)=\int_{-\infty}^{t} \exp (-\omega(t-r)) S(t-r) f(r) d r,
$$

where $\{S(t)\}_{t \geq 0}$ denotes the corresponding $C_{0}$-contraction semigroup. Moreover, a very general approach using a representation formula is given by Prüss [8] for linear Volterra-integrodifferential equations. In the nonlinear case, such formulas do not exist.

Recalling [6, 9], we learn that, for the nonlinear inhomogeneous Cauchy problem, almost periodic, asymptotically almost periodic, Eberlein weakly almost periodic, or $C_{0}$ right-hand sides will lead mainly under compactness conditions on the resolvent of $A$ and uniform convexity of the dual space to solutions of the same type.

In this paper, we discuss the problem in the following more general form. Given a closed and translation-invariant subspace, we investigate under which conditions on the nonlinearity $A$ a solution of the same type exists. The starting 
points of $[6,9]$ are the stability inequalities of the underlying differential equations. This limits the result to special assumptions on continuity properties of the norm and the range of the solution $u$ and the right-hand side $f$. In this paper, the solution is constructed by involving an adequate existence theory to the equation

$$
u^{\prime}(t)+A(u(t))+\omega u(t) \ni f(t), \quad t \in \mathbb{R},
$$

in a real Banach space $X$, with $\omega$ positive and $A m$-accretive in $X$.

The following are the main results of this paper. Let $Y$ be a closed and translation-invariant linear subspace of $\operatorname{BUC}(\mathbb{R}, X)$ of bounded and uniformly continuous functions from $\mathbb{R}$ into $X$. Assume that the resolvent $J_{\lambda}=(I+\lambda A)^{-1}$ of $A$ leaves $Y$ invariant, that is, if $h \in Y$, then the function $\left\{s \mapsto J_{\lambda} h(s)\right\}$ is in $Y$, for all $\lambda>0$. Then, we have the following:

(a) if $f \in Y$, then the integral solution $u$ to (1.2) (see Definition 5.1) is an element of $Y$ as well,

(b) $\lim _{t \rightarrow \infty}\|v(t)-u(t)\|=0$ for the integral solution of the initial value problem

$$
v^{\prime}(t)+A(v(t))+\omega v(t) \ni f(t), \quad t \geq 0, \quad v(0)=v_{0}
$$

$$
\text { for all } v_{0} \in \overline{D(A)} \text { (Theorem 5.2). }
$$

We note in passing that these results heavily depend on the assumption that $\omega>0$. For $\omega=0$, there are various counterexamples to the above inheritance property, see [5, Example 4.21] or [6, Example 5.2].

The main results will be applied to the particular cases $Y=A P(\mathbb{R}, X)$, the space of almost periodic functions and $Y=W(\mathbb{R}, X)$, the space of Eberlein weakly almost periodic functions, as well as to functions having a limit at infinity.

The main technique of the proof consists in showing that, for the case of $\omega>0$, the approximate solutions to (1.2), as considered by [2], converge to the integral solution of (1.2), not just locally but uniformly on all of $\mathbb{R}$ (Section 5). The necessary technical prerequisites are the subject of Sections 2, 3, and 4.

We finally note that, in the case of $A$ linear, the corresponding results on the asymptotic behaviour of the solutions to (1.2) can be derived even for the case $\omega=0$ if, instead, we invoke conditions on the relation of the spectra of $A$ and $f$ (cf. [8]). Thus, one of the points of our results here is that, in the nonlinear case-with no "spectrum" of $A$ available_-such spectral conditions may be compensated by adding the positive multiple $\omega I$ to $A$ in (1.2). For the existence of bounded solutions for history-dependent problems, we refer to Kartsatos [4]. 
Notation. (1) The bracket $[\cdot, \cdot]_{+}: X \times X \rightarrow \mathbb{R}$ is defined as the right-hand Gâteaux-derivative of the norm

$$
[x, y]_{+}:=\inf _{\lambda>0} \frac{\|x+\lambda y\|-\|x\|}{\lambda} .
$$

(2) We recall that $A \subset X \times X$ is accretive if and only if

$$
[x-\hat{x}, y-\hat{y}]_{+} \geq 0 \quad \forall(x, y),(\hat{x}, \hat{y}) \in A .
$$

An accretive operator is called $m$-accretive if $R(I+\lambda A)=X$ for all $\lambda>0$.

(3) Throughout this paper, $J_{\lambda}:=(I+\lambda A)^{-1}$ denotes the resolvent of $A$.

\section{Approximants}

Similar to the proof of existence given by [2], we consider the Yosida approximation of the linear part of the equation. Looking for solutions on the whole line, consider the following for $\lambda>0$ :

$$
\frac{1}{\lambda}\left(u(t)-\frac{1}{\lambda} \int_{0}^{\infty} \exp \left(-\frac{s}{\lambda}\right) u(t-s) d s\right)+A(u(t))+\omega u(t) \ni f(t), \quad t \in \mathbb{R} .
$$

For the approximations, we have the following proposition.

Proposition 2.1. Let $Y$ be a closed translation-invariant subspace of $\mathrm{BUC}(\mathbb{R}, X)$. If, for given $h \in Y$, the function $\left\{s \mapsto J_{\lambda}(h(s))\right\}$ is in $Y$, then (2.1) admits a solution $u \in Y$. Moreover, for given right-hand sides $f, g \in \mathrm{BUC}(\mathbb{R}, X)$, and corresponding solutions $u$ and $v$,

$$
\begin{aligned}
\|u(t)-v(t)\| \leq & \frac{\lambda}{1+\lambda \omega}\|f(t)-g(t)\| \\
& +\left(\frac{1}{1+\lambda \omega}\right) 2 \int_{0}^{\infty} \exp \left(-\frac{\omega \tau}{1+\lambda \omega}\right)\|f(t-\tau)-g(t-\tau)\| d \tau,
\end{aligned}
$$

and consequently,

$$
\begin{aligned}
\| u(t)- & u(t+h) \| \\
\leq & \frac{\lambda}{1+\lambda \omega}\|f(t)-f(t+h)\| \\
& +\left(\frac{1}{1+\lambda \omega}\right)^{2} \int_{0}^{\infty} \exp \left(-\frac{\omega \tau}{1+\lambda \omega}\right)\|f(t-\tau)-f(t+h-\tau)\| d \tau .
\end{aligned}
$$

Proof. Given $\lambda>0$, the solution to (2.1) will be found by applying the Banach fixed-point principle whereby the mapping

$$
F(u)(t):=J_{\lambda /(1+\lambda \omega)}\left(\frac{1}{1+\lambda \omega}\left(\lambda f(t)+\int_{0}^{\infty} \frac{1}{\lambda} \exp \left(-\frac{s}{\lambda}\right) u(t-s) d s\right)\right)
$$

is obtained by rewriting $(2.1)$. 
Comparing the solutions $u$ and $v$ for the right-hand sides $f$ and $g$, we get

$$
\begin{aligned}
\|u(t)-v(t)\|= & \| J_{\lambda /(1+\lambda \omega)}\left(\frac{1}{1+\lambda \omega}\left(\lambda f(t)+\int_{0}^{\infty} \frac{1}{\lambda} \exp \left(-\frac{s}{\lambda}\right) u(t-s) d s\right)\right) \\
& -J_{\lambda /(1+\lambda \omega)}\left(\frac{1}{1+\lambda \omega}\left(\lambda g(t)+\int_{0}^{\infty} \frac{1}{\lambda} \exp \left(-\frac{s}{\lambda}\right) v(t-s) d s\right)\right) \| \\
\leq & \frac{\lambda}{1+\lambda \omega}\|f(t)-g(t)\| \\
& +\frac{1}{1+\lambda \omega}\left\|\int_{0}^{\infty} \frac{1}{\lambda} \exp \left(-\frac{s}{\lambda}\right)(u(t-s)-v(t-s)) d s\right\| \\
\leq & \frac{\lambda}{1+\lambda \omega}\|f(t)-g(t)\| \\
& +\frac{1}{1+\lambda \omega} \int_{0}^{\infty} \frac{1}{\lambda} \exp \left(-\frac{s}{\lambda}\right)\|u(t-s)-v(t-s)\| d s .
\end{aligned}
$$

The solution to the integral equation

$$
u(t)=f(t)+\alpha \int_{0}^{\infty} \exp (-\beta \tau) u(t-\tau) d \tau
$$

for $0<\alpha<\beta$, is given by

$$
u(t)=(R f)(t):=f(t)+\alpha \int_{0}^{\infty} \exp (-(\beta-\alpha) \tau) f(t-\tau) d \tau
$$

Noting that the resolvent $R$ is positive, the above inequality yields

$$
\begin{aligned}
\|u(t)-v(t)\| \leq & \frac{\lambda}{1+\lambda \omega}\|f(t)-g(t)\| \\
& +\left(\frac{1}{1+\lambda \omega}\right)^{2} \int_{0}^{\infty} \exp \left(-\frac{\omega s}{1+\lambda \omega}\right)\|f(t-s)-g(t-s)\| d s .
\end{aligned}
$$

From Gripenberg [2, Theorem 1], we know that, for a given initial value $u_{0} \in$ $\overline{D(A)}$, the solutions $\left\{u_{\lambda}\right\}$ of

$$
\begin{gathered}
\frac{1}{\lambda}\left(u_{\lambda}(t)-u_{0}-\int_{0}^{t} \frac{1}{\lambda} \exp \left(-\frac{r}{\lambda}\right)\left(u_{\lambda}(t-r)-u_{0}\right) d r\right) \\
+A\left(u_{\lambda}(t)\right)+\omega u_{\lambda}(t) \ni f(t), \quad t>0
\end{gathered}
$$

will converge uniformly on compact sets to the so-called generalized solution of (1.3). For the connection between the solution on the whole axis (1.2) and the Cauchy problem (1.3), we provide two lemmas. 
Lemma 2.2. Let $f, g \in \mathrm{BUC}\left(\mathbb{R}^{+}, X\right)$, and let $u_{\lambda}, v_{\lambda}$ be the corresponding solutions to (2.9) with the initial values $u_{\lambda}^{0}, v_{\lambda}^{0}$. Then,

$$
\begin{aligned}
\left\|u_{\lambda}(t)-v_{\lambda}(t)\right\| \leq & \frac{\lambda}{1+\lambda \omega}\|f(t)-g(t)\| \\
& +\frac{1}{1+\lambda \omega} \exp \left(-\frac{\omega t}{1+\lambda \omega}\right)\left\|u_{\lambda}^{0}-v_{\lambda}^{0}\right\| \\
& +\left(\frac{1}{1+\lambda \omega}\right)^{2} \int_{0}^{t} \exp \left(-\frac{\omega r}{1+\lambda \omega}\right)\|f(t-r)-g(t-r)\| d r .
\end{aligned}
$$

Proof. From (2.9), we, after rearranging, obtain

$$
\begin{aligned}
u_{\lambda}(t)=J_{\lambda /(1+\lambda \omega)}\left(\frac{1}{1+\lambda \omega}(\right. & \lambda f(t)+\exp \left(-\lambda^{-1} t\right) u_{\lambda}^{0} \\
& \left.\left.+\int_{0}^{t} \frac{1}{\lambda} \exp \left(-\frac{s}{\lambda}\right) u_{\lambda}(t-s) d s\right)\right) .
\end{aligned}
$$

Hence,

$$
\begin{aligned}
\left\|u_{\lambda}(t)-v_{\lambda}(t)\right\| \leq & \frac{\lambda}{1+\lambda \omega}\|f(t)-g(t)\| \\
& +\frac{1}{1+\lambda \omega} \exp \left(-\lambda^{-1} t\right)\left\|u_{\lambda}^{0}-v_{\lambda}^{0}\right\| \\
& +\frac{1}{1+\lambda \omega} \int_{0}^{t} \frac{1}{\lambda} \exp \left(-\frac{s}{\lambda}\right)\left\|u_{\lambda}(t-s)-v_{\lambda}(t-s)\right\| d s .
\end{aligned}
$$

As in the previous proof, this time, using the generalized Gronwall lemma [3, page 257], we get

$$
\begin{aligned}
\left\|u_{\lambda}(t)-v_{\lambda}(t)\right\| \leq & \frac{\lambda}{1+\lambda \omega}\|f(t)-g(t)\|+\frac{1}{1+\lambda \omega} \exp \left(-\lambda^{-1} t\right)\left\|u_{\lambda}^{0}-v_{\lambda}^{0}\right\| \\
& +\frac{1}{(1+\lambda \omega)^{2}} \int_{0}^{t} \exp \left(-\frac{\omega(t-r)}{1+\lambda \omega}\right)\|f(r)-g(r)\| d r \\
& +\frac{1}{\lambda(1+\lambda \omega)^{2}} \int_{0}^{t} \exp \left(-\frac{\omega(t-r)}{1+\lambda \omega}\right) \exp \left(-\lambda^{-1} r\right) d r\left\|u_{\lambda}^{0}-v_{\lambda}^{0}\right\| \\
= & \frac{\lambda}{1+\lambda \omega}\|f(t)-g(t)\| \\
& +\frac{1}{1+\lambda \omega} \exp \left(-\frac{\omega t}{1+\lambda \omega}\right)\left\|u_{\lambda}^{0}-v_{\lambda}^{0}\right\| \\
& +\frac{1}{(1+\lambda \omega)^{2}} \int_{0}^{t} \exp \left(-\frac{\omega(t-r)}{1+\lambda \omega}\right)\|f(r)-g(r)\| d r .
\end{aligned}
$$


642 Bounded solutions of nonlinear Cauchy problems

Lemma 2.3. Let $f \in \operatorname{BUC}(\mathbb{R}, X), v_{0} \in \overline{D(A)}$, and let $u_{\lambda}$ be the solution of (2.1), and $v_{\lambda}$ be the solution of (2.9) with the initial value $v_{0}$. Then, for $t \geq 0$,

$$
\begin{aligned}
\left\|u_{\lambda}(t)-v_{\lambda}(t)\right\| \leq & \frac{1}{1+\lambda \omega} \exp \left(-\frac{\omega t}{1+\lambda \omega}\right)\left\|u_{\lambda}(0)-v_{0}\right\| \\
& +\frac{\omega}{1+\lambda \omega} \int_{-\infty}^{0} \exp \left(-\lambda^{-1}(t-r)\right)\left\|u_{\lambda}(r)-u_{\lambda}(0)\right\| d r \\
& +\frac{1}{1+\lambda \omega} \exp \left(-\frac{\omega t}{1+\lambda \omega}\right) \int_{-\infty}^{0} \frac{1}{\lambda} \exp \left(\lambda^{-1} r\right)\left\|u_{\lambda}(r)-u_{\lambda}(0)\right\| d r
\end{aligned}
$$

Proof. To give the connection between the solutions of (2.1) and (2.9), we rewrite (2.1) to obtain

$$
\begin{gathered}
\frac{1}{\lambda}\left(u_{\lambda}(t)-u_{\lambda}(0)-\frac{1}{\lambda} \int_{0}^{t} \exp \left(-\frac{t-r}{\lambda}\right)\left(u_{\lambda}(r)-u_{\lambda}(0)\right) d r\right)+A\left(u_{\lambda}(t)\right)+\omega u_{\lambda}(t) \\
\ni f(t)+\frac{1}{\lambda^{2}} \int_{-\infty}^{0} \exp \left(-\frac{t-r}{\lambda}\right)\left(u_{\lambda}(r)-u_{\lambda}(0)\right) d r .
\end{gathered}
$$

Thus, by Lemma 2.2, we find

$$
\begin{aligned}
\left\|u_{\lambda}(t)-v_{\lambda}(t)\right\| \leq & \frac{1}{\lambda(1+\lambda \omega)} \int_{-\infty}^{0} \exp \left(-\lambda^{-1}(t-r)\right)\left\|u_{\lambda}(r)-u_{\lambda}(0)\right\| d r \\
& +\frac{1}{1+\lambda \omega} \exp \left(-\frac{\omega t}{1+\lambda \omega}\right)\left\|u_{\lambda}(0)-v_{0}\right\| \\
& +\frac{1}{\lambda^{2}(1+\lambda \omega)^{2}} \int_{0}^{t} \exp \left(-\frac{\omega(t-r)}{1+\lambda \omega}\right) \\
= & \frac{\omega}{1+\lambda \omega} \int_{-\infty}^{0} \exp \left(-\lambda^{-1}(t-r)\right)\left\|u_{\lambda}(r)-u_{\lambda}(0)\right\| d r \\
& +\frac{1}{1+\lambda \omega} \exp \left(-\frac{\omega t}{1+\lambda \omega}\right)\left\|u_{\lambda}(0)-v_{0}\right\| \\
& +\frac{1}{1+\lambda \omega} \exp \left(-\frac{\omega t}{1+\lambda \omega}\right) \int_{-\infty}^{0} \frac{1}{\lambda} \exp \left(\lambda^{-1} r\right)\left\|u_{\lambda}(r)-u_{\lambda}(0)\right\| d r .
\end{aligned}
$$

\section{Integral solution}

In this section, we show that the generalized solution provided by Gripenberg [2] and the integral solution of (1.3) coincide. For the sake of completeness, we recall the definition of the integral solution. 
Definition 3.1. Let $x \in X$, let $f$ be a Bochner integrable function on $[0, T]$, and let $\omega$ be a real number. We call $u:[0, T] \rightarrow X$ an integral solution of type $\omega$ of the Cauchy problem (1.3) if

(1) $u(0)=x$;

(2) $u$ is continuous on $[0, T]$;

(3) for every $0<s<t<T$ and $(x, y) \in A+\omega I$, we have

$$
\begin{aligned}
\|u(t)-x\| \leq & \exp (-\omega(t-s))\|u(s)-x\| \\
& +\int_{s}^{t} \exp (-\omega(t-r))[u(r)-x, f(r)-y]_{+} d r .
\end{aligned}
$$

The next lemma will be the first step for showing that the generalized solution and the integral solution coincide.

Lemma 3.2. Let $f \in \mathrm{BUC}\left(\mathbb{R}^{+}, X\right)$ and let $u_{\lambda}$ be the solution of (2.9) with the initial value $u_{0}$, then

$$
\begin{aligned}
\left\|u_{\lambda}(t)-x\right\| \leq & \frac{\lambda}{1+\lambda \omega}\left[u_{\lambda}(t)-x, f(t)-y\right]_{+} \\
& +\frac{1}{1+\lambda \omega} \exp \left(-\frac{\omega t}{1+\lambda \omega}\right)\left\|u_{0}-x\right\| \\
& +\frac{1}{(1+\lambda \omega)^{2}} \int_{0}^{t} \exp \left(-\frac{\omega \tau}{1+\lambda \omega}\right)\left[u_{\lambda}(t-\tau)-x, f(t-\tau)-y\right]_{+} d \tau,
\end{aligned}
$$

for all $(x, y) \in A+\omega I$.

Proof. For $y \in A x+\omega x$, we, after rearranging, obtain

$$
x+\frac{\lambda}{1+\lambda \omega} A x \ni \frac{1}{1+\lambda \omega}\left(\lambda y+\exp \left(-\frac{t}{\lambda}\right) x+\frac{1}{\lambda} \int_{0}^{t} \exp \left(-\frac{r}{\lambda}\right) x d r\right) .
$$

Rearranging (2.9) gives

$$
\begin{aligned}
u_{\lambda}(t)+\frac{\lambda}{1+\lambda \omega} A\left(u_{\lambda}(t)\right) \ni \frac{1}{1+\lambda \omega}( & \lambda f(t)+\exp \left(-\frac{t}{\lambda}\right) u_{0} \\
& \left.+\frac{1}{\lambda} \int_{0}^{t} \exp \left(-\frac{r}{\lambda}\right) u_{\lambda}(t-r) d r\right) .
\end{aligned}
$$

As $A$ is accretive, we obtain

$$
\begin{gathered}
\left\|u_{\lambda}(t)-x\right\| \leq \frac{1}{1+\lambda \omega}\left(\lambda\left[u_{\lambda}(t)-x, f(t)-y\right]_{+}+\exp \left(-\frac{t}{\lambda}\right)\left\|u_{0}-x\right\|\right. \\
\left.+\frac{1}{\lambda} \int_{0}^{t} \exp \left(-\frac{r}{\lambda}\right)\left\|u_{\lambda}(t-r)-x\right\| d r\right)
\end{gathered}
$$


644 Bounded solutions of nonlinear Cauchy problems

The generalized Gronwall lemma leads to

$$
\begin{aligned}
\left\|u_{\lambda}(t)-x\right\| \leq \frac{1}{1+\lambda \omega}( & \lambda\left[u_{\lambda}(t)-x, f(t)-y\right]_{+}+\exp \left(-\frac{t}{\lambda}\right)\left\|u_{0}-x\right\| \\
& +\frac{1}{1+\lambda \omega} \int_{0}^{t} \exp \left(-\frac{\omega r}{1+\lambda \omega}\right)\left[u_{\lambda}(t-r)-x, f(t-r)-y\right]_{+} d r \\
& \left.+\frac{1}{1+\lambda \omega} \frac{1}{\lambda} \int_{0}^{t} \exp \left(-\frac{\omega r}{1+\lambda \omega}\right) \exp \left(-\frac{t-r}{\lambda}\right) d r\left\|u_{0}-x\right\|\right) \\
\leq \frac{1}{1+\lambda \omega}( & \lambda\left[u_{\lambda}(t)-x, f(t)-y\right]_{+} \\
& +\frac{1}{1+\lambda \omega} \int_{0}^{t} \exp \left(-\frac{\omega r}{1+\lambda \omega}\right)\left[u_{\lambda}(t-r)-x, f(t-r)-y\right]_{+} d r \\
& \left.+\exp \left(-\frac{\omega t}{1+\lambda \omega}\right)\left\|u_{0}-x\right\|\right) .
\end{aligned}
$$

Proposition 3.3. Let $u$ be the generalized solution of (1.3), with the right-hand side $f$ and the initial value $u_{0}$, let $u_{\lambda}$ be the solution of (2.9), for $h>0$, let $f_{h}(t):=$ $f(t+h), v_{0}:=u(h)$, and let $v_{\lambda}$ be the solution of

$$
\begin{gathered}
\frac{1}{\lambda}\left(v_{\lambda}(t)-v_{0}-\int_{0}^{t} \frac{1}{\lambda} \exp \left(-\frac{r}{\lambda}\right)\left(v_{\lambda}(t-r)-v_{0}\right) d r\right) \\
+A\left(v_{\lambda}(t)\right)+\omega v_{\lambda}(t) \ni f_{h}(t), \quad t>0,
\end{gathered}
$$

that is, $v_{\lambda}$ is the solution of (2.9) with the right-hand side $f_{h}$, and the initial value $v_{0}$.

Then,

(1) $\lim _{\lambda \rightarrow 0}\left\|v_{\lambda}(t)-u_{\lambda}(t+h)\right\|=0$ uniformly on compact sets, that is,

$$
\lim _{\lambda \rightarrow 0} v_{\lambda}(t)=u(t+h)
$$

(2) moreover,

$$
\begin{aligned}
\|u(t+h)-x\| \leq & \exp (-\omega t)\|u(h)-x\| \\
& +\int_{h}^{t+h} \exp (-\omega(t+h-r))[u(r)-x, f(r)-y]_{+} d r
\end{aligned}
$$

for all $(x, y) \in A+\omega I, u$ is consequently the integral solution of (1.3).

Proof. Rearranging (2.9) and (3.7) gives

$$
\begin{aligned}
& u_{\lambda}(t+h)+\frac{\lambda}{1+\lambda \omega} A\left(u_{\lambda}(t+h)\right) \\
& \ni \frac{1}{1+\lambda \omega}\left(\lambda f(t+h)+\exp \left(-\frac{t+h}{\lambda}\right) u_{0}\right. \\
& \left.\quad+\frac{1}{\lambda} \int_{0}^{t+h} \exp \left(-\frac{r}{\lambda}\right) u_{\lambda}(t+h-r) d r\right),
\end{aligned}
$$




$$
\begin{aligned}
v_{\lambda}(t)+ & \frac{\lambda}{1+\lambda \omega} A\left(v_{\lambda}(t)\right) \\
& \ni \frac{1}{1+\lambda \omega}\left(\lambda f(t+h)+\exp \left(-\frac{t}{\lambda}\right) u(h)+\frac{1}{\lambda} \int_{0}^{t} \exp \left(-\frac{r}{\lambda}\right) v_{\lambda}(t-r) d r\right) .
\end{aligned}
$$

By accretiveness of $A$, we obtain

$$
\begin{aligned}
\left\|u_{\lambda}(t+h)-v_{\lambda}(t)\right\| \leq \frac{1}{1+\lambda \omega}( & {\left[u_{\lambda}(t+h)-v_{\lambda}(t), \exp \left(-\frac{t+h}{\lambda}\right) u_{0}\right]_{+} } \\
& +\left[u_{\lambda}(t+h)-v_{\lambda}(t),\right. \\
& \frac{1}{\lambda} \int_{t}^{t+h} \exp \left(-\frac{r}{\lambda}\right) u_{\lambda}(t+h-r) d r \\
& \left.-\exp \left(-\frac{t}{\lambda}\right) u(h)\right]_{+} \\
& \left.+\frac{1}{\lambda} \int_{0}^{t} \exp \left(-\frac{r}{\lambda}\right)\left\|u_{\lambda}(t+h-r)-v_{\lambda}(t-r)\right\| d r\right) \\
\frac{1}{1+\lambda \omega}( & \exp \left(-\frac{t+h}{\lambda}\right)\left(\left\|u_{0}\right\|+\|u(h)\|\right) \\
& +\frac{1}{\lambda} \int_{t}^{t+h} \exp \left(-\frac{r}{\lambda}\right)\left\|u_{\lambda}(t+h-r)-u(h)\right\| d r \\
& \left.+\frac{1}{\lambda} \int_{0}^{t} \exp \left(-\frac{r}{\lambda}\right)\left\|u_{\lambda}(t+h-r)-v_{\lambda}(t-r)\right\| d r\right) .
\end{aligned}
$$

For given $\epsilon>0$, choose, by the local uniform convergence of the $u_{\lambda}$ and the continuity of $u, 0<\delta<h$ and $0<\lambda_{0}$ such that for all $0<\lambda<\lambda_{0}$,

$$
\sup _{0<s<\delta}\left\|u_{\lambda}(h-s)-u(h-s)\right\|+\sup _{0<s<\delta}\|u(h-s)-u(h)\|<\frac{\epsilon}{4}
$$

and, by the local boundedness of $u_{\lambda}$ and $u$,

$$
\exp \left(-\frac{\delta}{\lambda}\right)\left(\sup _{0 \leq s \leq h}\left\|u_{\lambda}(s)\right\|+\left\|u_{0}\right\|+2\|u(h)\|\right)<\frac{\epsilon}{4}
$$


646 Bounded solutions of nonlinear Cauchy problems

This choice gives

$$
\begin{aligned}
\frac{1}{\lambda} \int_{t}^{t+h} & \exp \left(-\frac{s}{\lambda}\right)\left\|u_{\lambda}(t+h-s)-u(h)\right\| d s \\
= & \frac{1}{\lambda} \int_{0}^{h} \exp \left(-\frac{s+t}{\lambda}\right)\left\|u_{\lambda}(h-s)-u(h)\right\| d s \\
= & \frac{1}{\lambda} \int_{0}^{\delta} \exp \left(-\frac{s+t}{\lambda}\right)\left\|u_{\lambda}(h-s)-u(h)\right\| d s \\
& +\frac{1}{\lambda} \int_{\delta}^{h} \exp \left(-\frac{s+t}{\lambda}\right)\left\|u_{\lambda}(h-s)-u(h)\right\| d s \\
\leq & \exp \left(-\frac{t}{\lambda}\right)\left(\sup _{0<s<\delta}\left\|u_{\lambda}(h-s)-u(h)\right\|\right. \\
& \left.\quad+\exp \left(-\frac{\delta}{\lambda}\right)\left(\sup _{0 \leq s \leq h}\left\|u_{\lambda}(s)\right\|+\|u(h)\|\right)\right) \\
\leq & \exp \left(-\frac{t}{\lambda}\right)\left(\frac{\epsilon}{4}+\exp \left(-\frac{\delta}{\lambda}\right)\left(\sup _{0 \leq s \leq h}\left\|u_{\lambda}(s)\right\|+\|u(h)\|\right)\right) .
\end{aligned}
$$

Thus,

$$
\begin{aligned}
\left\|u_{\lambda}(t+h)-v_{\lambda}(t)\right\| & \\
\leq \frac{1}{1+\lambda \omega}\left(\operatorname { e x p } ( - \frac { t } { \lambda } ) \left(\frac{\epsilon}{4}+\exp \left(-\frac{\delta}{\lambda}\right)\right.\right. & \left.\quad \times\left(\left\|u_{0}\right\|+2\|u(h)\|+\sup _{0 \leq s \leq h}\left\|u_{\lambda}(s)\right\|\right)\right) \\
& \left.\times \frac{1}{\lambda} \int_{0}^{t} \exp \left(-\frac{r}{\lambda}\right)\left\|u_{\lambda}(t+h-r)-v_{\lambda}(t-r)\right\| d r\right) .
\end{aligned}
$$

Now, an application of the generalized Gronwall lemma gives

$$
\begin{aligned}
& \left\|u_{\lambda}(t+h)-v_{\lambda}(t)\right\| \\
& \leq \frac{1}{1+\lambda \omega}\left(\exp \left(-\frac{t}{\lambda}\right)\left(\frac{\epsilon}{2}\right)+\frac{1}{\lambda(1+\lambda \omega)} \int_{0}^{t} \exp \left(-\frac{\omega(t-r)}{1+\lambda \omega}\right) \exp \left(-\frac{r}{\lambda}\right) d r\right) \frac{\epsilon}{2} \\
& \leq \epsilon
\end{aligned}
$$

Part (2) of Proposition 3.3 is a consequence of part (1) and Lemma 5.

Uniform convergence on $\mathbb{R}$ and $\mathbb{R}^{+}$, respectively, of the Yosida approximants $u_{\lambda}$ defined by (1.2) and (2.9) will be provided in Theorem 5.2 and Corollary 5.4. 


\section{Inequalities and lemmas}

Following the proof of Gripenberg by applying positive resolvents in a Banach lattice, we have to compute $\left(I-T_{\lambda, \mu}\right)^{-1}$ for

$$
\begin{aligned}
T_{\lambda, \mu} f(t, s):= & \frac{\mu}{\lambda+\mu+\lambda \mu \omega} \frac{1}{\lambda} \int_{0}^{\infty} \exp \left(-\frac{1}{\lambda} \tau\right) f(t-\tau, s) d \tau \\
& +\frac{\lambda}{\lambda+\mu+\lambda \mu \omega} \frac{1}{\mu} \int_{0}^{\infty} \exp \left(-\frac{1}{\mu} \tau\right) f(t, s-\tau) d \tau
\end{aligned}
$$

on $\operatorname{BUC}(\mathbb{R} \times \mathbb{R})$. For this aim, the modified Bessel functions $I_{0}$ and $I_{1}$ are needed

$$
\begin{gathered}
J(x, y):=I_{0}(2 \sqrt{\alpha \gamma x y})=\sum_{k=0}^{\infty} \frac{\alpha^{k} \gamma^{k} x^{k} y^{k}}{(k !)^{2}}=\frac{2}{\pi} \int_{0}^{1} \frac{1}{\sqrt{1-t^{2}}} \cosh (2 t \sqrt{\alpha \gamma x y}) d t, \\
I_{0}(0)=1,\left.\quad \partial_{x} I_{0}(2 \sqrt{\alpha \gamma x})\right|_{x=0}=0, \\
\partial_{x} I_{0}(2 \sqrt{\alpha \gamma x y})=\sqrt{\frac{\alpha \gamma y}{x}} I_{1}(2 \sqrt{\alpha \gamma x y}), \\
\partial_{x} \partial_{y} I_{0}(2 \sqrt{\alpha \gamma x y})=\alpha \gamma I_{0}(2 \sqrt{\alpha \gamma x y}) .
\end{gathered}
$$

The equations in Remark 4.1 are obtained by viewing the application of the reslovent to the function $\{t \mapsto 1\}$ as a Laplace transform. Moreover, it will be shown which parts of the kernel for the resolvent $T_{\lambda, \mu}$ are neglectable when $\lambda, \mu \rightarrow 0$.

Remark 4.1. The following identities [10, pages 208-209] will give a first insight into the behavior of the kernel if $(\lambda, \mu) \rightarrow 0$ :

$$
\begin{gathered}
\frac{\lambda}{\lambda+\mu+\lambda \mu \omega} \int_{0}^{\infty} \int_{0}^{\infty}\left(\partial_{1} J\right)(x, y) \exp \left(-\frac{(1+\lambda \omega) y+(1+\mu \omega) x}{\lambda+\mu+\lambda \mu \omega}\right) d y d x \\
=\frac{\lambda(\lambda+\mu+\lambda \mu \omega)}{(1+\mu \omega) \omega}, \\
\frac{\mu}{\lambda+\mu+\lambda \mu \omega} \int_{0}^{\infty} \int_{0}^{\infty}\left(\partial_{2} J\right)(x, y) \exp \left(-\frac{(1+\lambda \omega) y+(1+\mu \omega) x}{\lambda+\mu+\lambda \mu \omega}\right) d y d x \\
\frac{\mu(\lambda+\mu+\lambda \mu \omega)}{(1+\lambda \omega) \omega}, \\
\left.\frac{(1+\lambda \omega) y+(1+\mu \omega) x}{\lambda+\mu+\lambda \mu \omega}\right) d y d x \\
=\frac{2}{\omega(\lambda+\mu+\lambda \mu \omega)} .
\end{gathered}
$$


648 Bounded solutions of nonlinear Cauchy problems

The next lemma provides a representation for the positive resolvent of the operator $T_{\lambda, \mu}$.

Lemma 4.2. For the operator $T_{\lambda, \mu}$ defined above,

(1) $\left\|T_{\lambda, \mu}\right\| \leq(\lambda+\mu) /(\lambda+\mu+\lambda \mu \omega)<1$, consequently,

$$
\left\|\left(I-T_{\lambda, \mu}\right)^{-1}\right\| \leq \frac{\lambda+\mu+\lambda \mu \omega}{\lambda \mu \omega}
$$

and $\left(I-T_{\lambda, \mu}\right)^{-1}$ is positive;

(2) letting

$$
\alpha=\frac{\mu}{\lambda+\mu+\lambda \mu \omega} \frac{1}{\lambda}, \quad \gamma=\frac{\lambda}{\lambda+\mu+\lambda \mu \omega} \frac{1}{\mu},
$$

the resolvent is given by

$$
\begin{aligned}
\left(I-T_{\lambda, \mu}\right)^{-1} u(t, s) & \\
= & u(t, s)+\gamma \int_{0}^{\infty} \exp \left(-\frac{(1+\lambda \omega) y}{\lambda+\mu+\lambda \mu \omega}\right) u(t, s-y) d y \\
& +\alpha \int_{0}^{\infty} \exp \left(-\frac{(1+\mu \omega) x}{\lambda+\mu+\lambda \mu \omega}\right) u(t-x, s) d x \\
& +\gamma \int_{0}^{\infty} \int_{0}^{\infty} \partial_{x} I_{0}(2 \sqrt{\alpha \gamma x y}) \exp \left(-\frac{(1+\lambda \omega) y+(1+\mu \omega) x}{\lambda+\mu+\lambda \mu \omega}\right) \\
& +\alpha \int_{0}^{\infty} \int_{0}^{\infty} \partial_{y} I_{0}(2 \sqrt{\alpha \gamma x y}) \exp \left(-\frac{(1+\lambda \omega) y+(1+\mu \omega) x}{\lambda+\mu+\lambda \mu \omega}\right) \\
& \times u(t-x, s-y) d y d x \\
+ & 2 \alpha \gamma \int_{0}^{\infty} \int_{0}^{\infty} I_{0}(2 \sqrt{\alpha \gamma x y}) \exp \left(-\frac{(1+\lambda \omega) y+(1+\mu \omega) x}{\lambda+\mu+\lambda \mu \omega}\right) \\
& \times u(t-x, s-y) d y d x .
\end{aligned}
$$

Proof. Defining

$$
\beta:=\frac{1}{\lambda}, \quad \delta:=\frac{1}{\mu},
$$


we have to find a bounded solution of the integral equation

$$
\begin{aligned}
y(t, s) & -\alpha \int_{-\infty}^{t} \exp (-\beta(t-\tau)) y(\tau, s) d \tau \\
& -\gamma \int_{-\infty}^{s} \exp (-\delta(s-\sigma)) y(t, \sigma) d \sigma=f(t, s) .
\end{aligned}
$$

For $f \in C_{b}^{2}(\mathbb{R} \times \mathbb{R})$, we define

$$
g(t, s):=\exp (-\alpha t-\gamma s) \partial_{t} \partial_{s}(\exp (\beta t+\delta s) f(t, s))
$$

The solution $b(t, s)$ to the wave equation

$$
\partial_{1} \partial_{2} b(t, s)-\alpha \gamma b(t, s)=\exp (-\alpha t-\gamma s) \partial_{1} \partial_{2}(\exp (\beta t+\delta s) f(t, s))=: g(t, s)
$$

such that, for a constant $C>0$,

$$
|b(t, s)| \leq C \exp ((\beta-\alpha) t+(\delta-\gamma) s)
$$

holds, is given by [10, pages 68-69]

$$
\begin{aligned}
b(t, s)= & \int_{-\infty}^{t} \int_{-\infty}^{s} I_{0}(2 \sqrt{\alpha \gamma(t-x)(s-y)}) g(x, y) d x d y \\
= & \int_{0}^{\infty} \int_{0}^{\infty} I_{0}(2 \sqrt{\alpha \gamma x y}) \exp (-\alpha(t-x)-\gamma(s-y)) \partial_{1} \partial_{2} \\
& \quad \times(\exp (\beta(t-x)+\delta(s-y)) f(t-x, s-y)) d x d y .
\end{aligned}
$$

To enlarge the domain of the solution operator, we will rewrite the representation formula by partial integration. For this purpose, let

$$
\begin{aligned}
& B f(t, s):=\int_{0}^{\infty} \int_{0}^{\infty} J(x, y) \exp (-\alpha(t-x)-\gamma(s-y)) f(t-x, s-y) d x d y \\
& B_{i} f(t, s):=\int_{0}^{\infty} \int_{0}^{\infty} \partial_{i} J(x, y) \exp (-\alpha(t-x)-\gamma(s-y)) f(t-x, s-y) d x d y .
\end{aligned}
$$


The following identities hold for the integral operators $B$ and $B_{i}$ :

$$
\begin{aligned}
B\left(\partial_{1} f\right) & (t, s) \\
= & \int_{0}^{\infty} \int_{0}^{\infty} J(x, y) \exp (-\alpha(t-x)-\gamma(s-y))\left(-\partial_{x}\right) f(t-x, s-y) d x d y \\
= & -\left.\int_{0}^{\infty} J(x, y) \exp (-\alpha(t-x)-\gamma(s-y)) f(t-x, s-y) d y\right|_{0} ^{\infty} \\
& +B_{1} f(t, s)+\alpha B f(t, s) \\
= & \exp (-\alpha t) \int_{0}^{\infty} \exp (-\gamma(s-y)) f(t, s-y) d y \\
& +B_{1} f(t, s)+\alpha B f(t, s), \\
B\left(\partial_{2} f\right) & (t, s) \\
= & \int_{0}^{\infty} \int_{0}^{\infty} J(x, y) \exp (-\alpha(t-x)-\gamma(s-y))\left(-\partial_{y}\right) f(t-x, s-y) d x d y \\
= & -\left.\int_{0}^{\infty} J(x, y) \exp (-\alpha(t-x)-\gamma(s-y)) f(t-x, s-y) d x\right|_{0} ^{\infty} \\
& +B_{2} f(t, s)+\gamma B f(t, s) \\
= & \exp (-\gamma s) \int_{0}^{\infty} \exp (-\alpha(t-x)) f(t-x, s) d x \\
& +B_{2} f(t, s)+\gamma B f(t, s),
\end{aligned}
$$$$
B_{1}\left(\partial_{2} f\right)(t, s)
$$$$
=\int_{0}^{\infty} \int_{0}^{\infty}\left(\partial_{1} J\right)(x, y) \exp (-\alpha(t-x)-\gamma(s-y))
$$$$
\times\left(-\partial_{y}\right) f(t-x, s-y) d x d y
$$$$
=-\left.\int_{0}^{\infty}\left(\partial_{1} J\right)(x, y) \exp (-\alpha(t-x)-\gamma(s-y)) f(t-x, s-y) d x\right|_{0} ^{\infty}
$$$$
+\alpha \gamma B f(t, s)+\gamma B_{1} f(t, s)
$$$$
=+\alpha \gamma B f(t, s)+\gamma B_{1} f(t, s),
$$$$
B\left(\partial_{1} \partial_{2}\left(\exp \left(\beta(\cdot)_{1}+\delta(\cdot)_{2}\right) f\right)\right)(t, s)
$$

$$
\begin{aligned}
= & \exp ((\beta-\alpha) t) \int_{0}^{\infty} \exp (-\gamma(s-y))\left(-\partial_{y}\right) \\
& \times(\exp (\delta(s-y)) f(t, s-y)) d y \\
& +B_{1}\left(\partial_{2}\left(\exp \left(\beta(\cdot)_{1}+\delta(\cdot)_{2}\right) f\right)\right)(t, s) \\
& +\alpha B\left(\partial_{2}\left(\exp \left(\beta(\cdot)_{1}+\delta(\cdot)_{2}\right) f\right)\right)(t, s)
\end{aligned}
$$




$$
\begin{aligned}
= & \exp ((\beta-\alpha) t) \int_{0}^{\infty} \exp (-\gamma(s-y))\left(-\partial_{y}\right)(\exp (\delta(s-y)) f(t, s-y)) d y \\
& +\alpha \gamma B\left(\exp \left(\beta(\cdot)_{1}+\delta(\cdot)_{2}\right) f\right)(t, s)+\gamma B_{1}\left(\exp \left(\beta(\cdot)_{1}+\delta(\cdot)_{2}\right) f\right)(t, s) \\
& +\alpha \exp ((\delta-\gamma) s) \int_{0}^{\infty} \exp ((\beta-\alpha)(t-x)) f(t-x, s) d x \\
& +\alpha B_{2}\left(\exp \left(\beta(\cdot)_{1}+\delta(\cdot)_{2}\right) f\right)(t, s)+\alpha \gamma B\left(\exp \left(\beta(\cdot)_{1}+\delta(\cdot)_{2}\right) f\right)(t, s) \\
= & \exp ((\beta-\alpha) t+(\delta-\gamma) s) f(t, s) \\
& +\gamma \exp ((\beta-\alpha) t) \int_{0}^{\infty} \exp ((\delta-\gamma)(s-y)) f(t, s-y) d y \\
& +\alpha \gamma B\left(\exp \left(\beta(\cdot)_{1}+\delta(\cdot)_{2}\right) f\right)(t, s)+\gamma B_{1}\left(\exp \left(\beta(\cdot)_{1}+\delta(\cdot)_{2}\right) f\right)(t, s) \\
& +\alpha \exp ((\delta-\gamma) s) \int_{0}^{\infty} \exp ((\beta-\alpha)(t-x)) f(t-x, s) d x \\
& +\alpha B_{2}\left(\exp \left(\beta(\cdot)_{1}+\delta(\cdot)_{2}\right) f\right)(t, s)+\alpha \gamma B\left(\exp \left(\beta(\cdot)_{1}+\delta(\cdot)_{2}\right) f\right)(t, s) \\
= & \exp ((\beta-\alpha) t+(\delta-\gamma) s) f(t, s) \\
& +\gamma \exp ((\beta-\alpha) t) \int_{0}^{\infty} \exp ((\delta-\gamma)(s-y)) f(t, s-y) d y \\
& +\alpha \exp ((\delta-\gamma) s) \int_{0}^{\infty} \exp ((\beta-\alpha)(t-x)) f(t-x, s) d x \\
& +\gamma B_{1}\left(\exp \left(\beta(\cdot)_{1}+\delta(\cdot)_{2}\right) f\right)(t, s) \\
& +\alpha B_{2}\left(\exp \left(\beta(\cdot)_{1}+\delta(\cdot)_{2}\right) f\right)(t, s) \\
& +2 \alpha \gamma B\left(\exp \left(\beta(\cdot)_{1}+\delta(\cdot)_{2}\right) f\right)(t, s) .
\end{aligned}
$$

The substitution $a(t, s)=\exp (\alpha t+\gamma s) b(t, s)$ will provide a solution to the differential equation

$$
\partial_{1} \partial_{2} a(t, s)-\alpha \partial_{2} a(t, s)-\gamma \partial_{1} a(t, s)=\partial_{1} \partial_{2}(\exp (\beta t+\delta s) f(t, s)),
$$

and, for a constant $C>0$ and $i=1,2$,

$$
\left|\left(\partial_{i} a\right)(t, s)\right|,|a(t, s)| \leq C \exp (\beta t+\delta s) .
$$

Integration of the differential equation will lead to the integral equation

$$
a(t, s)-\alpha \int_{-\infty}^{t} a(\tau, s) d \tau-\gamma \int_{-\infty}^{s} a(t, \sigma) d \sigma=\exp (\beta t+\delta s) f(t, s) .
$$

Fitting the equations together, we obtain for

$$
y(t, s):=\exp (-\beta t-\delta s) a(t, s)=\exp ((\alpha-\beta) t+(\gamma-\delta) s) b(t, s)
$$


652 Bounded solutions of nonlinear Cauchy problems

that

$$
\begin{aligned}
y(t, s)= & \left(I-T_{\lambda, \mu}\right)^{-1} f(t, s)=f(t, s) \\
& +\gamma \int_{0}^{\infty} \exp ((\gamma-\delta) y) f(t, s-y) d y \\
& +\alpha \int_{0}^{\infty} \exp ((\alpha-\delta) x) f(t-x, s) d x \\
& +\gamma \int_{0}^{\infty} \int_{0}^{\infty}\left(\partial_{1} J\right)(x, y) \exp ((\alpha-\beta) x+(\gamma-\delta) y) f(t-x, s-y) d y d x \\
& +\alpha \int_{0}^{\infty} \int_{0}^{\infty}\left(\partial_{2} J\right)(x, y) \exp ((\alpha-\beta) x+(\gamma-\delta) y) f(t-x, s-y) d y d x \\
& +2 \alpha \gamma \int_{0}^{\infty} \int_{0}^{\infty} J(x, y) \exp ((\alpha-\beta) x+(\gamma-\delta) y) f(t-x, s-y) d y d x
\end{aligned}
$$

and thus the desired resolvent.

For the next lemma due to Crandall and Evans, we fit the positive $\omega$ into their original proof.

Lemma 4.3 [1]. Let $x_{1}, x_{2}, y_{1}, y_{2}, f, g \in X, \delta, \gamma, \omega>0, A$ accretive, and

$$
\frac{x_{1}-x_{2}}{\gamma}+A x_{1}+\omega x_{1} \ni f, \quad \frac{y_{1}-y_{2}}{\delta}+A y_{1}+\omega y_{1} \ni g
$$

Then,

$$
\left\|x_{1}-y_{1}\right\| \leq \frac{\gamma \delta}{\gamma+\delta+\gamma \delta \omega}\left(\left[x_{1}-y_{1}, f-g\right]_{+}+\gamma^{-1}\left\|x_{2}-y_{1}\right\|+\delta^{-1}\left\|x_{1}-y_{2}\right\|\right) .
$$

Proof. By accretiveness of $A$,

$$
\begin{aligned}
\omega\left\|x_{1}-y_{1}\right\| \leq & {\left[x_{1}-y_{1},\left(f+\frac{x_{2}-x_{1}}{\gamma}\right)-\left(g+\frac{y_{2}-y_{1}}{\delta}\right)\right]_{+} } \\
\leq & {\left[x_{1}-y_{1}, f-g\right]+\gamma^{-1}\left[x_{1}-y_{1}, x_{2}-y_{1}\right]_{+}+\delta^{-1}\left[x_{1}-y_{1}, y_{1}-y_{2}\right]_{+} } \\
\leq & {\left[x_{1}-y_{1}, f-g\right]_{+}+\gamma^{-1}\left(\left\|x_{2}-y_{1}\right\|-\left\|x_{1}-y_{1}\right\|\right) } \\
& +\delta^{-1}\left(\left\|x_{1}-y_{2}\right\|-\left\|x_{1}-y_{1}\right\|\right) .
\end{aligned}
$$

Rearranging concludes the proof. 


\section{Main results}

For the solution on the whole real line, we introduce the following notion.

Definition 5.1. Let $f \in L^{\infty}(\mathbb{R}, X)$ and let $\omega$ be a real number. A function $u \in$ $\operatorname{BUC}(\mathbb{R}, X)$ is called an integral solution of type $\omega$ of (1.2) if, for all $h \in \mathbb{R}, u(h) \in$ $\overline{D(A)}$ and

$$
v(t):=u(t+h)
$$

is an integral solution of type $\omega$ to

$$
\begin{gathered}
v^{\prime}(t)+A v(t)+\omega v(t) \ni f(t+h), \quad t>0, \\
v(0)=u(h) .
\end{gathered}
$$

The next theorem will be the key to all our results.

TheOrem 5.2. Let $Y$ be a closed translation-invariant subspace of $\mathrm{BUC}(\mathbb{R}, X)$, and let $f \in Y$. If, for given $h \in Y$ and $\lambda>0$, the function $\left\{s \mapsto J_{\lambda}(h(s))\right\}$ is in $Y$, then (1.2) admits an integral solution $u$ of type $\omega$ on $\mathbb{R}$. Furthermore, the solution $u$ belongs to $Y$. For every integral solution $v$ of (1.3),

$$
\|v(t)-u(t)\| \leq \exp (-\omega t)\left\|u(0)-v_{0}\right\|, \quad t \geq 0 .
$$

For given right-hand sides $f, g \in \mathrm{BUC}(\mathbb{R}, X)$ and the corresponding integral solutions $u$, $v$ of (1.2),

$$
\|u(t)-v(t)\| \leq \int_{0}^{\infty} \exp (-\omega \tau)\|f(t-\tau)-g(t-\tau)\| d \tau
$$

for all $t \in \mathbb{R}$.

Proof. In the first part, we show the uniform convergence on $\mathbb{R}$ of the approximants $u_{\lambda}$ to a function $u \in Y$. In the second part, we prove that $u$ fulfills the definition of an integral solution on $\mathbb{R}$.

For given $\lambda>0$, we have

$$
\frac{1}{\lambda}\left(u_{\lambda}(t)-\int_{0}^{\infty} \frac{1}{\lambda} \exp \left(-\frac{s}{\lambda}\right) u_{\lambda}(t-s) d s\right)+\omega u_{\lambda}(t)+A\left(u_{\lambda}(t)\right) \ni f(t) .
$$

Thus, for pairs $\left(u_{\lambda}(t), \lambda\right)$ and $\left(u_{\mu}(s), \mu\right)$, an application of the inequality given by Lemma 4.3 implies

$$
\begin{aligned}
\left\|u_{\lambda}(t)-u_{\mu}(s)\right\| \leq \frac{1}{\lambda+\mu+\lambda \mu \omega}\{ & \lambda \mu\left[u_{\lambda}(t)-u_{\mu}(s), f(t)-f(s)\right]_{+} \\
& +\frac{\mu}{\lambda} \int_{0}^{\infty} \exp \left(\frac{-\tau}{\lambda}\right)\left\|u_{\lambda}(t-\tau)-u_{\mu}(s)\right\| d \tau \\
& \left.+\frac{\lambda}{\mu} \int_{0}^{\infty} \exp \left(\frac{-\tau}{\mu}\right)\left\|u_{\lambda}(t)-u_{\mu}(s-\tau)\right\| d \tau\right\} .
\end{aligned}
$$


Using the resolvent computed in Lemma 4.2 and Remark 4.1, it remains to consider the last part of the kernel. Moreover, to prove uniform convergence, only the case $t=s$ has to be considered.

We define $\beta:=\lambda+\mu+\lambda \mu \omega$, let $\delta>0$, and let $\epsilon_{f}>0$ be the corresponding modulus of uniform continuity for $f$, and apply Lemma $4.2(1)$ to obtain

$$
\begin{aligned}
& \frac{2 \mu \lambda}{\beta^{3}} \int_{0}^{\infty} \int_{0}^{\infty} I_{0}\left(2 \sqrt{\frac{x y}{\beta^{2}}}\right) \\
& \quad \times \exp \left(-\frac{(1+\lambda \omega) y+(1+\mu \omega) x}{\beta}\right)\|f(t-x)-f(t-y)\| d y d x \\
& \leq \frac{1}{\omega} \sup _{|x-y|<\delta}\|f(x)-f(y)\| \\
& +\frac{2 \mu \lambda}{\beta^{3}} \int_{x, y \geq 0,|x-y|>\delta}\left(2 \sqrt{\frac{x y}{\beta^{2}}}\right) \\
& \quad \times \exp \left(-\frac{(1+\lambda \omega) y+(1+\mu \omega) x}{\beta}\right)\|f(t-x)-f(t-y)\| d(y, x) \\
& \leq \frac{1}{\omega} \epsilon_{f}+\frac{4\|f\|_{\infty} \mu \lambda}{\beta^{3}} \int_{x, y \geq 0,|x-y|>\delta} I_{0}\left(2 \sqrt{\frac{x y}{\beta^{2}}}\right) \\
& \quad \times \exp \left(-\frac{(1+\lambda \omega) y+(1+\mu \omega) x}{\beta}\right) d(y, x) .
\end{aligned}
$$

Consequently, it remains to discuss the integral on a closed set complementary to a strip containing the diagonal. Therefore, we will use the integral representation of the Bessel function (cf. [7, page 159])

$$
I_{0}\left(2 \sqrt{\frac{x y}{\beta^{2}}}\right)=\frac{2}{\pi} \int_{0}^{1} \frac{1}{\sqrt{1-t^{2}}} \cosh \left(2 t \sqrt{\frac{x y}{\beta^{2}}}\right) d t .
$$

The estimation has to be done on

$$
\{(x, y): x \geq \delta, 0 \leq y \leq x-\delta\} \cup\{(x, y): x \geq 0, x+\delta \leq y\}
$$

As the domain is symmetric with respect to the diagonal whereby the symmetry will interchange the roles $(x, y)$ and $(\lambda, \mu)$, it remains to consider the integral on half of the domain. For this aim, we substitute

$$
r=\frac{x}{\beta}, \quad s=\frac{y}{\beta} .
$$


Fitting these together, we obtain

$$
\begin{aligned}
\frac{\lambda \mu}{\beta^{3}} \int_{0}^{\infty} & \int_{x+\delta}^{\infty} I_{0}\left(2 \sqrt{\frac{x y}{\beta^{2}}}\right) \exp \left(-\frac{(1+\lambda \omega) y+(1+\mu \omega) x}{\beta}\right) d(y, x) \\
= & \frac{\lambda \mu}{\beta} \int_{0}^{\infty} \int_{\delta / \beta+r}^{\infty} I_{0}(2 \sqrt{r s}) \exp (-(1+\lambda \omega) s-(1+\mu \omega) r) d s d r \\
\leq & \frac{2 \lambda \mu}{\beta} \int_{0}^{\infty} \int_{\delta / \beta+r}^{\infty} \exp (2 \sqrt{r s}) \exp (-(1+\lambda \omega) s-(1+\mu \omega) r) d s d r \\
\leq & \frac{2 \lambda \mu}{\beta} \int_{0}^{\beta^{-3 / 2}} \int_{\delta / \beta+r}^{\infty} \exp \left(-(\sqrt{s}-\sqrt{r})^{2}\right) \exp (-\omega(\lambda r+\mu s)) d s d r \\
& +\frac{2 \lambda \mu}{\beta} \int_{\beta^{-3 / 2}}^{\infty} \int_{\delta / \beta+r}^{\infty} \exp (-\omega(\lambda r+\mu s)) d s d r
\end{aligned}
$$

For $0 \leq r \leq \beta^{-3 / 2}$ and $\delta \sqrt{\beta} \leq 3$, the inequality

$$
\begin{aligned}
\sqrt{s}-\sqrt{r} & \geq \sqrt{\frac{\delta}{\beta}+r}-\sqrt{r} \geq \frac{\delta}{\sqrt{\beta}} \frac{1}{\sqrt{\delta+\beta r}+\sqrt{\beta r}} \\
& \geq \frac{\delta}{\sqrt[4]{\beta}} \frac{1}{\sqrt{(\sqrt{\beta} \delta+1)}+1} \geq \frac{\delta}{3 \sqrt[4]{\beta}}
\end{aligned}
$$

leads to

$$
\exp \left(-(\sqrt{s}-\sqrt{r})^{2}\right) \leq \exp \left(-\frac{\delta^{2}}{9 \sqrt{\beta}}\right)
$$

which gives

$$
\begin{aligned}
\frac{\lambda \mu}{\beta} \int_{0}^{\beta^{-3 / 2}} & \int_{\delta / \beta+r}^{\infty} \exp \left(-(\sqrt{s}-\sqrt{r})^{2}\right) \exp (-\omega(\lambda r+\mu s)) d s d r \\
& \leq \frac{\lambda \mu}{\beta} \exp \left(-\frac{\delta^{2}}{9 \sqrt{\beta}}\right) \int_{0}^{\beta^{-3 / 2}} \int_{\delta / \beta+r}^{\infty} \exp (-\omega(\lambda r+\mu s)) d s d r \\
& =\frac{\lambda}{\beta \omega} \exp \left(-\frac{\delta^{2}}{9 \sqrt{\beta}}\right) \int_{0}^{\beta^{-3 / 2}} \exp \left(-\frac{\omega \mu \delta}{\beta}-\omega(\lambda+\mu) r\right) d r \\
& \leq \frac{1}{\omega^{2}} \frac{\lambda}{\lambda+\mu} \frac{1}{\beta} \exp \left(-\frac{\delta^{2}}{9 \sqrt{\beta}}\right) \exp \left(-\frac{\omega \mu \delta}{\beta}\right) .
\end{aligned}
$$

Thus, the first integral will converge to zero. 
For the remaining term, we have

$$
\begin{gathered}
\frac{\lambda \mu}{\beta} \int_{\beta^{-3 / 2}}^{\infty} \int_{\delta / \beta+r}^{\infty} \exp \left(-(\sqrt{s}-\sqrt{r})^{2}\right) \exp (-\omega(\lambda r+\mu s)) d s d r \\
\quad \leq \frac{\lambda \mu}{\beta} \int_{\beta^{-3 / 2}}^{\infty} \int_{\delta / \beta+r}^{\infty} \exp (-\omega(\lambda r+\mu s)) d s d r \\
\quad=\frac{1}{\omega^{2}} \frac{\lambda}{\lambda+\mu} \exp \left(-\frac{\omega \mu \delta}{\beta}\right) \frac{1}{\beta} \exp \left(-\omega \frac{\lambda+\mu}{\beta} \frac{1}{\sqrt{\beta}}\right) .
\end{gathered}
$$

This proves the uniform convergence of the approximants.

Thus, it remains to prove that $u$ fulfills Definition 5.1. Let $h \in \mathbb{R}$ and $v(t):=$ $u(t+h)$. As $u_{\lambda}(h) \in D(A)$ for all $\lambda>0, \lim _{\lambda \rightarrow 0} u_{\lambda}(h) \in \overline{D(A)}$. The translation invariance of (2.1) gives that $v_{\lambda}(t)$ is the solution of (2.1) with the right-hand side $f_{h}:=f(t+h)$.

Let $w=\lim _{\lambda \rightarrow 0} w_{\lambda}$ uniformly on compact sets of $\mathbb{R}^{+}$, where $w_{\lambda}$ is the solution to

$$
\begin{gathered}
\frac{1}{\lambda}\left(w_{\lambda}(t)-w_{0}-\int_{0}^{t} \frac{1}{\lambda} \exp \left(-\frac{r}{\lambda}\right)\left(w_{\lambda}(t-r)-w_{0}\right) d r\right) \\
+A\left(w_{\lambda}(t)\right)+\omega w_{\lambda}(t) \ni f_{h}(t), \quad t>0, \\
w_{0}=u(h) .
\end{gathered}
$$

From Lemma 2.3, we obtain

$$
\begin{aligned}
\| v_{\lambda}(t) & -w_{\lambda}(t) \| \\
\leq & \frac{1}{1+\lambda \omega} \exp \left(-\frac{\omega t}{1+\lambda \omega}\right)\left\|v_{\lambda}(0)-w_{0}\right\| \\
& +\frac{\omega}{1+\lambda \omega} \int_{-\infty}^{0} \exp \left(-\lambda^{-1}(t-r)\right)\left\|v_{\lambda}(r)-v_{\lambda}(0)\right\| d r \\
& +\frac{1}{1+\lambda \omega} \exp \left(-\frac{\omega t}{1+\lambda \omega}\right) \int_{-\infty}^{0} \frac{1}{\lambda} \exp \left(\lambda^{-1} r\right)\left\|v_{\lambda}(r)-v_{\lambda}(0)\right\| d r
\end{aligned}
$$

Since $w_{0}=u(h)=v(0)=\lim _{\lambda \rightarrow 0} v_{\lambda}(h)$, the first term tends to zero. For the second term, the Hölder inequality applies. Thus, it remains to consider the third 
term. Let $\epsilon>0$ and $\delta:=\delta(\epsilon)$ the modulus of continuity for $v$, then

$$
\begin{aligned}
\int_{-\infty}^{0} \frac{1}{\lambda} \exp \left(\lambda^{-1} r\right)\left\|v_{\lambda}(r)-v_{\lambda}(0)\right\| d r \\
=\int_{-\infty}^{-\delta} \frac{1}{\lambda} \exp \left(\lambda^{-1} r\right)\left\|v_{\lambda}(r)-v_{\lambda}(0)\right\| d r \\
\quad+\int_{-\delta}^{0} \frac{1}{\lambda} \exp \left(\lambda^{-1} r\right)\left\|v_{\lambda}(r)-v_{\lambda}(0)\right\| d r \\
\leq 2 \exp \left(-\frac{\delta}{\lambda}\right)\left\|v_{\lambda}\right\|_{\infty}+\sup _{0 \leq r \leq \delta}\left\|v_{\lambda}(r)-v_{\lambda}(0)\right\| .
\end{aligned}
$$

Which proves that $v=w$ is the integral solution.

Applying Proposition 2.1 with $h=-t$, we obtain

$$
\begin{aligned}
\left\|u_{\lambda}(t)-u_{\lambda}(0)\right\| \leq & \frac{\lambda}{1+\lambda \omega}\|f(t)-f(0)\| \\
& +\frac{1}{(1+\lambda \omega)^{2}} \int_{0}^{\infty} \exp \left(-\frac{\omega r}{1+\lambda \omega}\right)\|f(t-r)-f(-r)\| d r
\end{aligned}
$$

Lemma 2.3 leads to

$$
\begin{aligned}
\left\|u_{\lambda}(t)-v_{\lambda}(t)\right\| \leq & \frac{1}{1+\lambda \omega} \exp \left(-\frac{\omega t}{1+\lambda \omega}\right)\left\|u_{\lambda}(0)-v_{0}\right\| \\
& +\frac{\lambda \omega}{(1+\lambda \omega)^{2}} \int_{-\infty}^{0} \exp \left(-\lambda^{-1}(t-r)\right)\|f(r)-f(0)\| d r \\
& +\frac{\lambda}{(1+\lambda \omega)^{2}} \exp \left(-\frac{\omega t}{1+\lambda \omega}\right) \int_{-\infty}^{0} \frac{1}{\lambda} \exp \left(\lambda^{-1} s\right)\|f(s)-f(0)\| d s \\
& +\frac{\lambda \omega}{(1+\lambda \omega)^{3}} \int_{-\infty}^{0} \frac{1}{\lambda} \exp \left(-\lambda^{-1}(t-r)\right) \\
& +\frac{1}{(1+\lambda \omega)^{3}} \exp \left(-\frac{\omega t}{1+\lambda \omega}\right) \\
& \times \int_{0}^{\infty} \exp \left(-\frac{\omega r}{1+\lambda \omega}\right) \int_{-\infty}^{0} \frac{1}{\lambda} \exp \left(\lambda^{-1} s\right)\|f(s-r)-f(-r)\| d s d r .
\end{aligned}
$$


Except for the last integral, an application of the Hölder inequality applies. Thus, it remains to consider

$$
\begin{aligned}
\int_{-\infty}^{0} \frac{1}{\lambda} & \exp \left(\lambda^{-1} s\right)\|f(s-r)-f(-r)\| d s \\
\leq & \sup _{r \in \mathbb{R}} \sup _{0 \leq s \leq \delta}\|f(s-r)-f(-r)\| \\
& \quad+\int_{-\infty}^{-\delta} \frac{1}{\lambda} \exp \left(\lambda^{-1} s\right)\|f(s-r)-f(-r)\| d s,
\end{aligned}
$$

which gives, for fixed $t>0$ in the limit $\lambda \rightarrow 0$,

$$
\|u(t)-v(t)\| \leq \exp (-\omega t)\left\|u(0)-v_{0}\right\|
$$

From the uniform convergence of the approximants, inequality (5.4) becomes a consequence of Lebesgue's dominated convergence theorem and Proposition 2.1 .

Corollary 5.3. Let $f \in \operatorname{BUC}(\mathbb{R}, X)$, then the integral solution of $(1.2)$ is unique.

Proof. Let $u$ be an integral solution of (1.2), and $\hat{u}$ the solution of (1.2) found by the approximation through (2.1). As $A$ is assumed to be $m$-accretive, $v(t):=$ $u(t+h)$ is a mild solution of type $\omega$ to

$$
\begin{gathered}
v^{\prime}(t)+A(v(t))+\omega v(t) \ni f(t+h), \quad t \in \mathbb{R}^{+}, \\
v(0)=u(h),
\end{gathered}
$$

and $\hat{v}(t):=\hat{u}(t+h)$ is a mild solution of type $\omega$ to

$$
\begin{gathered}
\hat{v}^{\prime}(t)+A(\hat{v}(t))+\omega \hat{v}(t) \ni f(t+h), \quad t \in \mathbb{R}^{+}, \\
\hat{v}(0)=\hat{u}(h) .
\end{gathered}
$$

Consequently,

$$
\begin{aligned}
\|u(s)-\hat{u}(s)\| & =\|v(s-h)-\hat{v}(s-h)\| \\
& \leq \exp (-\omega(s-h))\|v(0)-\hat{v}(0)\| \\
& \leq \exp (-\omega(s-h))\|u(h)-\hat{u}(h)\| \\
& \longrightarrow 0,
\end{aligned}
$$

as $h \rightarrow-\infty$.

Corollary 5.4. Let $f \in \operatorname{BUC}\left(\mathbb{R}^{+}, X\right), u_{0} \in X$, and let $\left\{u_{\lambda}\right\}_{\lambda>0}$ be the solution to (2.9), and $u$ the integral solution to (1.3). Then,

$$
u_{\lambda} \rightarrow u \text { uniformly on }[0, \infty)
$$




\section{Applications}

Applying the norm continuity of the resolvent, we obtain Corollary 6.1 as a first consequence.

COROLlary 6.1. (1) Let the range of the right-hand side $f$ be relatively compact. Then, the range of the integral solution of the initial value problem (1.3) is relatively compact.

(2) If $f_{\infty}:=\lim _{t \rightarrow \infty} f(t)$, or $f_{-\infty}:=\lim _{t \rightarrow-\infty} f(t)$ exist, the same limits exist for the solution of (1.2) and are equal to $(A+\omega)^{-1}\left(f_{\infty}\right)$, or $(A+\omega)^{-1}\left(f_{-\infty}\right)$.

We give an extension of a result of Seifert [9], who proves the existence of an almost periodic integral solution for an almost periodic right-hand side.

Theorem 6.2 [9]. Let $f \in A P(\mathbb{R}, X)$. Then, there exists an almost periodic integral solution of (1.2).

In [6], Eberlein weakly almost periodic functions, that is, $f \in \operatorname{BUC}\left(\mathbb{R}^{+}, X\right)$ such that

$$
O(f):=\left\{f_{t}:=\{r \longmapsto f(t+r)\}: t \in \mathbb{R}^{+}\right\}
$$

is weakly relatively compact in $\mathrm{BUC}\left(\mathbb{R}^{+}, X\right)$, are considered. For these right-hand sides the, Eberlein weak almost periodicity of integral solutions is extended from the uniform convexity of the dual to the demicontinuity of $A$.

Corollary 6.3. Let $f$ be Eberlein weakly almost periodic, and let either $A$ be demicontinuous and $J_{\lambda}$ compact, or $f\left(\mathbb{R}^{+}\right)$be relatively compact. Then, the solution of the initial value problem (1.3) is Eberlein weakly almost periodic.

Proof. For proving the Eberlein weak almost periodicity of $\left\{t \mapsto J_{\lambda}(f(t))\right\}$, we apply [5, Theorem 2.3 and Corollary 2.5]; hence, it remains to prove weak-norm continuity of $J_{\lambda}$ on the closure of the range of $f$, in the case where $A$ is demicontinuous and $J_{\lambda}$ is compact. As $f\left(\mathbb{R}^{+}\right)$is weakly relatively compact in a separable subspace, it is enough to prove weak-norm sequential continuity. For a given $\left\{x_{n}\right\}_{n \in \mathbb{N}} \subset X, x_{n} \rightarrow x$ weakly, the compactness of $J_{\lambda}$ provides an element $u \in X$, and for a subsequence $\left\{x_{n_{l}}\right\}_{l \in \mathbb{N}}$ of $\left\{x_{n}\right\}_{n \in \mathbb{N}}$, a subsequence of $\left\{x_{n_{k}}\right\}_{k \in \mathbb{N}}$ such that

$$
u_{k}:=J_{\lambda}\left(x_{n_{l_{k}}}\right) \longrightarrow u,
$$

that is, $x_{n_{l_{k}}} \in u_{k}+\lambda A\left(u_{k}\right)$, and the demicontinuity leads to $J_{\lambda}(x)=u$. Consequently, $u$ is the unique limit, and the proof is complete.

We note in passing that every $f \in W(\mathbb{R}, X)$, and thus every $f \in A P(\mathbb{R}, X)$ as well is uniformly ergodic, that is, there exists $x_{f} \in X$ such that

$$
\limsup _{T \rightarrow \infty}\left\|\frac{1}{T} \int_{h}^{T+h} f(\tau) d \tau-x_{f}\right\|=0 .
$$


Obviously, our main result Theorem 5.2 can as well be applied to the space $Y$ of all uniformly ergodic functions from $\mathbb{R}$ into $X$. However, for the case of $A$ nonlinear, it seems hard to find conditions on $A$ or its resolvent $J_{\lambda}$ such that $J_{\lambda}$ leaves this space invariant.

The solution provided in Theorem 5.2 may be used to obtain an extension result. On $Y$, the derivative is $m$-accretive, and a condition is given when an extension of the operator

$$
\frac{d}{d t}+A(\cdot)
$$

on $Y$ is $m$-accretive. Let

$$
\begin{array}{r}
D_{Y}(B):=\{u \in Y: \\
: \exists f \in Y \text { s.t. } u \text { is a integral solution } \\
\text { on } \left.\mathbb{R} \text { to } u^{\prime}(t)+A(u(t)) \ni f(t)\right\} .
\end{array}
$$

For $u \in D_{Y}(B)$, define

$$
\begin{aligned}
B u:=\{f \in Y: & u \text { is a integral solution } \\
& \text { on } \left.\mathbb{R} \text { to } u^{\prime}(t)+A(u(t)) \ni f(t), \forall t \in \mathbb{R}\right\} .
\end{aligned}
$$

COROLlary 6.4. Let $Y$ be a closed translation-invariant linear subspace of $\operatorname{BUC}(\mathbb{R}, X)$. Then the operator $B \subset Y \times Y$ defined by (6.5) and (6.6) is m-accretive if, for all $f \in Y$, and $\lambda>0$, the function $\left\{t \mapsto J_{\lambda}^{A}(f(t))\right\}$ is in $Y$.

Proof. We start with proving $R(I+\lambda B)=Y$. That is, for given $f \in Y$, we have to look for a mild solution to

$$
u^{\prime}(t)+A(u(t))+\frac{1}{\lambda} u(t) \ni \frac{1}{\lambda} f(t) .
$$

By Theorem 5.2, we find a unique mild solution $u \in Y$, consequently, $u \in D_{Y}(B)$.

Therefore, it remains to prove the accretiveness of the operator $B$. For this aim, we prove that $J_{\lambda}^{B}$ is a contraction. As the resolvent is given by the solution found in Theorem 5.2, we have

$$
\begin{aligned}
& \left\|J_{\lambda}^{B}(f)(t)-J_{\lambda}^{B}(g)(t)\right\| \\
& \quad \leq \frac{1}{\lambda} \int_{0}^{\infty} \exp \left(-\frac{s}{\lambda}\right)\|f(t-s)-g(t-s)\| d s \\
& \quad \leq\|f-g\|_{\infty},
\end{aligned}
$$

which concludes the proof.

\section{Acknowledgment}

The author is grateful to the referee for his valuable conceptual suggestions. 


\section{References}

[1] M. G. Crandall and L. C. Evans, On the relation of the operator $\partial / \partial s+\partial / \partial \tau$ to evolution governed by accretive operators, Israel J. Math. 21 (1975), no. 4, 261-278.

[2] G. Gripenberg, Volterra integro-differential equations with accretive nonlinearity, J. Differential Equations 60 (1985), no. 1, 57-79.

[3] G. Gripenberg, S.-O. Londen, and O. Staffans, Volterra Integral and Functional Equations, Encyclopedia of Mathematics and Its Applications, vol. 34, Cambridge University Press, Cambridge, 1990.

[4] A. G. Kartsatos, The existence of bounded solutions on the real line of perturbed nonlinear evolution equations in general Banach spaces, Nonlinear Anal. 17 (1991), no. 11, 1085-1092.

[5] J. Kreulich, Eberlein weak almost periodicity and differential equations in Banach spaces, Ph.D. thesis, Universität Essen, Germany, 1992.

[6] Eberlein-weakly almost-periodic solutions of evolution equations in Banach spaces, Differential Integral Equations 9 (1996), no. 5, 1005-1027.

[7] N. N. Lebedew, Spezielle Funktionen und ihre Anwendungen, Bibliographisches Institut, Mannheim, 1973 (German).

[8] J. Prüss, Bounded solutions of Volterra equations, SIAM J. Math. Anal. 19 (1988), no. 1, 133-149.

[9] G. Seifert, Nonlinear evolution equations with almost periodic time dependence, SIAM J. Math. Anal. 18 (1987), no. 2, 387-392.

[10] D. Voelker and G. Doetsch, Die zweidimensionale Laplace-Transformation. Eine Einführung in ihre Anwendung zur Lösung von Randwertproblemen nebst Tabellen von Korrespondenzen, Lehrbücher und Monographien aus dem Gebiete der Exakten Wissenschaften, Mathematische Reihe, vol. 12, Verlag Birkhäuser, Basel, 1950 (German).

Josef Kreulich: FB Mathematik, Universität Essen, D-45117 Essen, Germany

E-mail address: josef.kreulich@t-online.de 


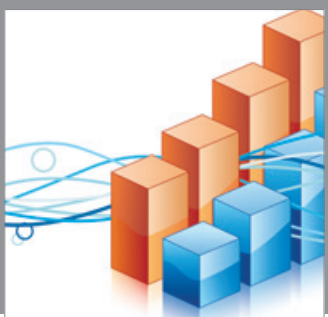

Advances in

Operations Research

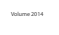

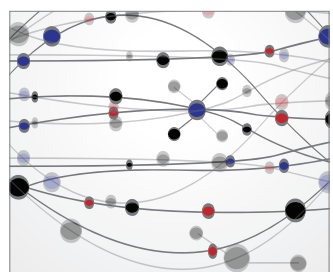

\section{The Scientific} World Journal
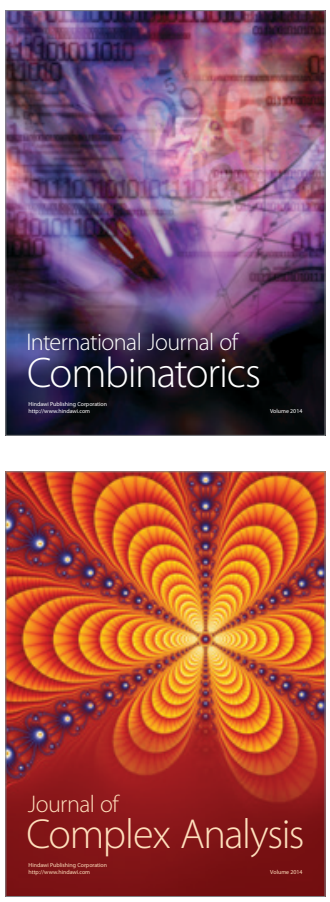

International Journal of

Mathematics and

Mathematical

Sciences
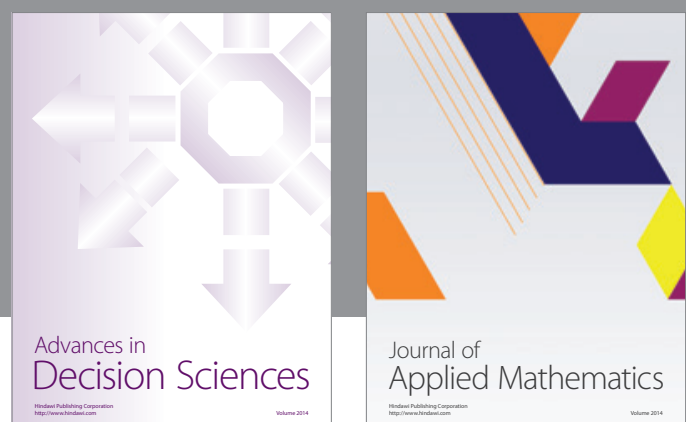

Journal of

Applied Mathematics
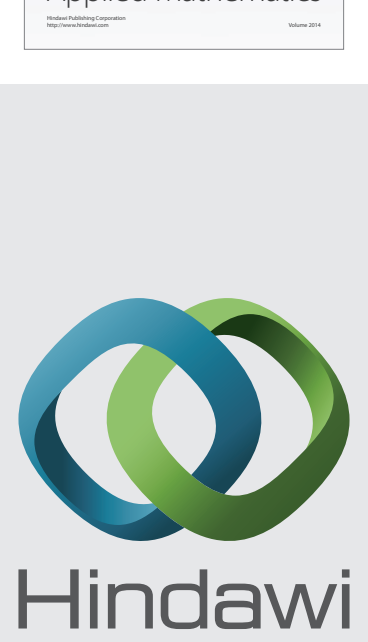

Submit your manuscripts at http://www.hindawi.com
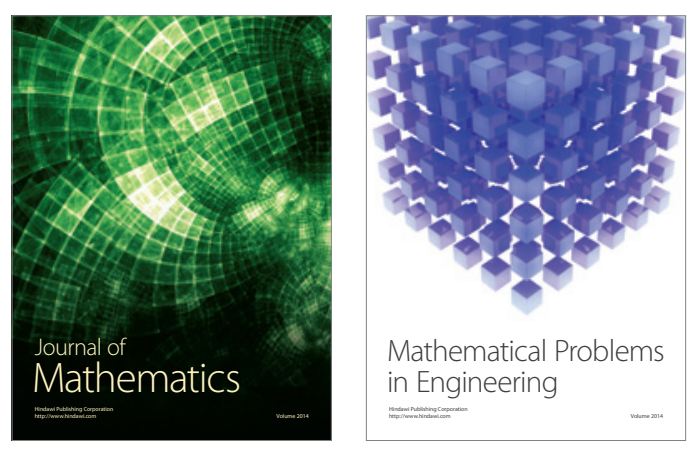

Mathematical Problems in Engineering
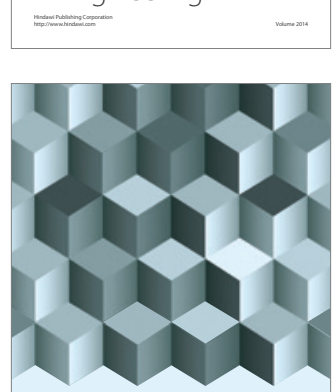

Journal of

Function Spaces
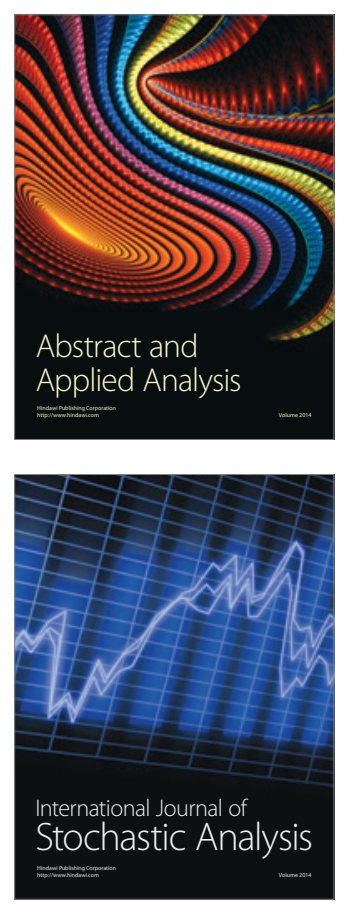

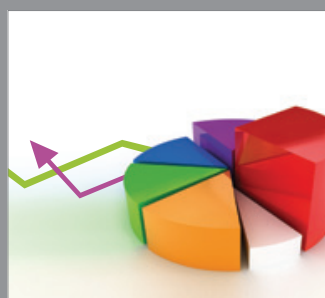

ournal of

Probability and Statistics

Promensencen
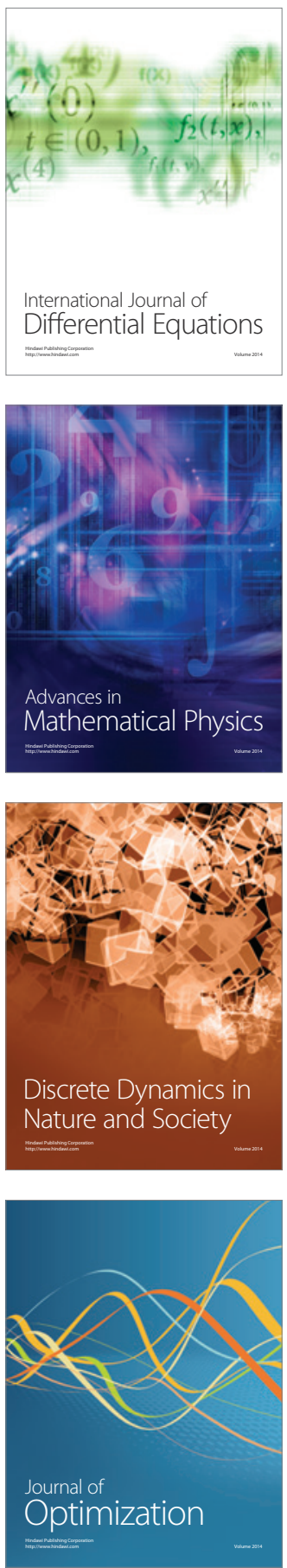